\title{
Testing quantum entanglement with local measurement
}

\author{
Qing Xie ${ }^{1,2 *}$, X.-X. Wu ${ }^{2}$, X.-M. Ding ${ }^{3}$, W.-L. Yang ${ }^{4}$, R.-H. Yue ${ }^{1}$, and Heng Fan ${ }^{2 \dagger}$ \\ ${ }^{1}$ Faculty of Science, Ningbo University, Ningbo, 315211, China \\ ${ }^{2}$ Institute of Physics, Chinese Academy of Sciences, Beijing 100190, China \\ ${ }^{3}$ Institute of Applied Mathematics, Academy of Mathematics and System Science, Chinese Academy of Sciences, Beijing 100190, China \\ ${ }^{4}$ Institute of Modern Physics, Northwest University, Xi'an 710069, China
}

(Dated: February 5, 2018)

\begin{abstract}
We propose to detect quantum entanglement by a condition of local measurments. We find that this condition can detect efficiently the pure entangled states for both discrete and continuous variable systems. It does not depend on interference of decoherence from noise and detection loss in some systems, which allows a loopholefree test in real experiments. In particular, it is a necessary condition for the violation of some generalized Bell inequalities.
\end{abstract}

PACS numbers: 03.65.Ud, 03.65.Ta, 42.50.Dv

\section{INTRODUCTION}

Quantum mechanics (QM) predicts correlations that no local realistic theory can ever reproduce. Local realistic theory states that there are hidden variables which determine the properties of physical system prior to and independent of measurement; also another assumption states that these properties of one system can not influenced by one another when these two systems are spacelike separated, which means spacelike separated systems have their own hidden variables, i.e. position and momentum in phase space.

Bell [2, 3] proposed his theorem that QM violates certain correlation function inequalities that any local hidden variable (LHV) theory must satisfy. This finding directly displays the incompatibility between QM and LHV theory for the first time. After Bell's seminal work, multiple Bell-type inequalities have been derived [4-8] and Bell inequality violations have been observed experimentally [9-12]. Nowadays, it is well known that the correlations QM imposed on outcomes of measurements carried out on spacelike separated systems are strictly larger than LHV model does.

In quantum information processing, entanglement plays a key role and is the invaluable resource. It is expected that the entangled state shared by spatially separated parties will violate some Bell inequalities [16]. On the other hand, if we restrict to pure states, we may propose some equalities based on local measurements which is much simpler than the Bell inequalities. These equalities is based on the fact that a separable pure state is simply the product state, so local measurements will not affect the measurements on other spatially separated parties. In this paper, we will apply this method to study the entanglement. We remark that this method is closely related with Bell inequality as we will show later, it was used to derive the Bell inequalities by Mermin for multipartite entangled states [5]. Also it is closely related with contextuality

\footnotetext{
*xieqingnbu@163.com

†hfan@iphy.ac.cn
}

[13, 14], as is well-known that QM is non-contextual, here our measurement is performed locally.

If one of those equal relations is infringed, we can conclude that the state under consideration is entangled. In practice, to observe a violation in real experiment, one may choose appropriate quantum system and measurement settings to make sure the two sides of these equality as different as possible to fight the interference of docoherence. This method can be applied on both discrete and continuous variable (CV) quantum systems. The application of CV case are not only of importance from the fundamental point of view, but also may be a applicable candidate to provide a loophole-free test. We present an explicit example to demonstrate that these correlation function equality are effective both for discrete and continuous variable system. Explicitly, any two qubit pure entangled state violate this equality, for discrete spin operators measurement and continuous quadrature operators measurement. The interference of decoherence from noise and detection loss in real experiment always decrease the possibility to observe a violation of Bell-type inequality which increases the difficulty and complexity to design an experimental scheme. In comparison, these interference from noise and detection loss decrease both side of our correlation function equality at the same rate. As we define the degree of violation by the ratio of two side of these equality, this quantity is unaffect by docoherence which make our equality particularly suitable for designing a loophole-free test. Particularly, we demonstrate that the general form of correlation function equality being violated is responsible for the violation of Cavalcanti-ForsterReid-Drummond (CFRD) inequality, a Bell-type inequality for CV system proposed recently [15]. Thus experimentally there is no need to measure all the commeasurable operators to observe a violation of CFRD inequality, only the responsible term alone can lead to a conclusion.

The paper is organized as follows: In section II, we propose the method and show that it can be applied to test entangled state. In section III, we apply this method to CV system. In section IV, we show that the violation of of those equality is responsible for the violation of CFRD inequality. In section $\mathrm{V}$, we extend the previous work of CFRD inequality to more 
general case. Section VI is a brief conclusion.

\section{CORRELATION FUNCTION EQUALITIES FOR SEPARABLE PURE STATES}

Let's first introduce the Bell-type inequality and the notations. The simplest correlation function inequality might be the Clauser-Horne-Shimony-Holt (CHSH) Bell-type scenario [4]: two space-separated observers, called Alice and Bob, each of them can choose between two dichotomic observables of two outcomes, labeled by \pm 1 . The $\mathrm{CHSH}$ inequality then reads

$$
E_{11}+E_{12}+E_{21}-E_{22} \leq 2
$$

where the functions $E_{i j}=\left\langle A_{i} B_{j}\right\rangle$, which are expectation values of Alice's observable choice $A_{i}$ and Bob's observable choice $B_{j}, i, j \in\{1,2\}$, known as correlation functions. For convenience we also use $A_{i}\left(B_{j}\right)$ denoting the outcome of Alice's (Bob's) measurement. It is well known that (1) holds for any LHV model. While in QM description, $A_{i}$ and $B_{j}$ are measurement operators with eigenvalues \pm 1 which can generally be written as $A_{i}=\mathbf{a}_{i} \cdot \boldsymbol{\sigma}$ and $B_{j}=\mathbf{b}_{j} \cdot \boldsymbol{\sigma}$, where $\boldsymbol{\sigma}=\left(\sigma_{x}, \sigma_{y}, \sigma_{z}\right)$ are Pauli matrices and $\mathbf{a}_{i}, \mathbf{b}_{j}$ are real unity vectors. Quantum entangled state violates $\mathrm{CHSH}$ inequality. For example, choosing local measurement $A_{1}=\sigma_{x}, A_{2}=$ $\sigma_{y}$ for Alice and $B_{1}=\sigma_{x}, B_{2}=\sigma_{y}$ for Bob, then $\mathrm{CHSH}$ inequality is maximally violated when system is prepared in maximally entangled state, $\left(|00\rangle+e^{i \phi}|11\rangle\right) / \sqrt{2}$.

One important assumption of LHV theory is that Alice's measurement on her own system can not influence Bob's when these two systems are spacelike separated. Then the above four correlation functions satisfy the following condition for any pure state with a product form, i.e., for a separable state,

$$
E_{i j} E_{k l}=E_{i l} E_{k j}
$$

where $i, j, k, l \in\{1,2\}$. Here thus we propose to use those equation as the detection of entangled states. Suppose system is prepared as a pure separable state, $\rho=\rho_{A} \otimes \rho_{B}$, it is obvious that,

$$
E_{i j}=\operatorname{tr}\left(A_{i} \rho_{A}\right) \operatorname{tr}\left(B_{j} \rho_{B}\right)
$$

Thus Eq.2 surely should be satisfied for any separable pure state.

We next consider a simple example of a two qubit entangled state, $|\Psi\rangle=\sin \theta|00\rangle+\cos \theta e^{i \phi}|11\rangle$. If Alice and Bob choose four measurement operators given above, then we have,

$$
\begin{aligned}
& E_{11} E_{22}=\left\langle\sigma_{x} \sigma_{x}\right\rangle\left\langle\sigma_{y} \sigma_{y}\right\rangle=-\sin ^{2} 2 \theta \cos ^{2} \phi \\
& E_{12} E_{21}=\left\langle\sigma_{x} \sigma_{y}\right\rangle\left\langle\sigma_{y} \sigma_{x}\right\rangle=\sin ^{2} 2 \theta \sin ^{2} \phi
\end{aligned}
$$

Equality holds only for, $\sin ^{2} 2 \theta=0$, this means that any pure entangled state violates (2). This is consistent with Gisin's theorem [16, 17]. Here relation (2) can determine completely whether this pure state is entangled or not.

Eq. (2) holds for any completely separable mixed state $\rho=$ $\rho_{A} \otimes \rho_{B}$, however, the general separable mixed state does not satisfy this condition. Suppose two-qubit mixed separable state have the form $\rho=\sum_{m} p_{m} \rho_{A}^{m} \otimes \rho_{B}^{m}$, where $\sum_{m} p_{m}=1$. This gives,

$$
\begin{aligned}
E_{i j} E_{k l} & =\sum_{m n} p_{m} p_{n} \operatorname{tr}\left(A_{i} \rho_{A}^{m}\right) \operatorname{tr}\left(B_{j} \rho_{B}^{m}\right) \operatorname{tr}\left(A_{k} \rho_{A}^{n}\right) \operatorname{tr}\left(B_{l} \rho_{B}^{n}\right), \\
E_{i l} E_{k j} & =\sum_{m n} p_{m} p_{n} \operatorname{tr}\left(A_{i} \rho_{A}^{m}\right) \operatorname{tr}\left(B_{l} \rho_{B}^{m}\right) \operatorname{tr}\left(A_{k} \rho_{A}^{n}\right) \operatorname{tr}\left(B_{j} \rho_{B}^{n}\right) .
\end{aligned}
$$

Generally, equality (2) is violated by mixed separable state, we may still need turn to Bell inequalities.

\section{APPLICATION TO CV}

We next consider the application of Eq. (2) to a CV system. Let us introduce the notations, defining quadrature operators $A_{1}=\hat{a}_{1}+\hat{a}_{1}^{\dagger}, A_{2}=i\left(\hat{a}_{1}-\hat{a}_{1}^{\dagger}\right)$ for Alice and $B_{1}=\hat{a}_{2}+\hat{a}_{2}^{\dagger}, B_{2}=-i\left(\hat{a}_{2}-\hat{a}_{2}^{\dagger}\right)$ for Bob, where $\hat{a}_{i}$ and $\hat{a}_{i}^{\dagger}$ are boson annihilation and creation operators. A simple example is that when system is prepared in a superposition of single photon state, $|\Psi\rangle=\sin \theta|01\rangle+\cos \theta e^{i \phi}|10\rangle$, where we have used the standard Fock-state representation, $|n\rangle_{A(B)} \equiv$ $\frac{\left(\hat{a}_{1(2)}^{\dagger}\right)^{n}}{\sqrt{n !}}|0\rangle_{A(B)}$, we obtain $E_{11} E_{22}=-\sin ^{2} 2 \theta \cos ^{2} \phi$ and $E_{12} E_{21}=\sin ^{2} 2 \theta \sin ^{2} \phi$. Eq.(2) is only satisfied when the quantum state is separable.

We consider the CV system as the two modes squeeze process which represents a nonlinear interaction of two quantized modes in a nonlinear medium with a strong classical pump field. The entangled state can be realized as the limiting case of a properly normalized two mode squeezed state (TMSS) for infinitely large squeezing. Explicitly, the TMSS takes the form,

$$
|\mathrm{TMSS}\rangle=e^{r\left(a_{1}^{\dagger} a_{2}^{\dagger}-a_{1} a_{2}\right)}|00\rangle=\sum_{n=0}^{\infty} \frac{\tanh ^{n} r}{\cosh r}|n n\rangle .
$$

By using the quadrature operators defined above, we can calculate that,

$$
\begin{aligned}
& E_{11} E_{22}=4\left(\sum_{n=0}^{\infty} \frac{\tanh ^{2 n+1} r}{\cosh ^{2} r}(n+1)\right)^{2} / \sum_{n=0}^{\infty} \frac{\tanh ^{2 n} r}{\cosh ^{2} r} \\
& E_{12} E_{21}=0
\end{aligned}
$$

Eq. (2) is not satisfied regardless of squeezing parameter $r,(r>0)$. On the other hand, we already know, the TMSS violates the Bell inequality for any $r,(r>0)$, see for example Ref.[18]. Of course, the degree of violation is still related with $r$. Thus Eq.(2) can be used to detect whether the involved state is entangled or not for both discrete and CV systems.

Though the proposed relation is not satisfied by general mixed state, it is, however, can be applied for case of mixed 
state constructed by a pure state with depolarizing noise. Let us start from the single photon entangled state, $|\Psi\rangle=$ $\sin \theta|01\rangle+\cos \theta e^{i \phi}|10\rangle$. Taking the interference of whitenoise like decoherence into account, the system is now in state,

$$
\rho=p|\Psi\rangle\langle\Psi|+(1-p) \frac{I}{\operatorname{tr} I},
$$

where $p$ is the probability that the state is unaffected by noise. We find $E_{11} E_{22}=-p^{2} \sin ^{2} 2 \theta \cos ^{2} \phi$ and $E_{12} E_{21}=$ $p^{2} \sin ^{2} 2 \theta \sin ^{2} \phi$. Then $p^{2}$ becomes an overall factor in both side of equality (2). If the degree of violation is defined by the ratio of r.h.s. and 1.h.s. of Eq.(2), it is unaffected by this depolarizing noise. Note that when $p$ becomes small, the state itself becomes separable [19]. Similar as for case of TMSS in (6), we consider state, $\rho=p|T M S S\rangle\langle T M S S|+(1-p) \frac{I}{\operatorname{tr} I}$. By straightforward calculation, we can also find $p^{2}$ as an overall factor in both sides of Eq. (2).

The importance of this property can be as follows. We know that the main loophole in experimental observation of Bell inequality is caused by the detection loss. Also it is known that the photon-detection with efficiency $\eta$ is practically equivalent to the ideal detection after the signal $\hat{a}$ is mixed with a vacuum $\hat{v}$ at a beam splitter of transmissivity $\sqrt{\eta}$. Namely, the observed signal $\hat{a}_{o}$ is expressed by $\hat{a}_{o}=$ $\sqrt{\eta} \hat{a}+\sqrt{1-\eta} \hat{v}$. This gives $E_{11} E_{22}=-\eta^{2} \sin ^{2} 2 \theta \cos ^{2} \phi$ and $E_{12} E_{21}=\eta^{2} \sin ^{2} 2 \theta \sin ^{2} \phi$ for single photon entangled state $|\Psi\rangle=\sin \theta|01\rangle+\cos \theta e^{i \phi}|10\rangle$. Thus by using the balanced homodyne detection to measure the quadrature amplitudes at each mode, $\eta^{2}$ becomes an overall factor in both sides, which makes our scheme insensitive to detector efficiency $\eta$. Thus the relation (2) can detect the entangled state of CV effectively.

\section{GENERALIZATION FOR MULTIPARTITE STATE AND CFRD INEQUALITY}

The correlation function equality (2) can be generalized to multipartite system straightforwardly by increasing the number of parties. Consider $n$ spatially separated observers and each of them can choose between $m$ experimental settings. The $i_{j}$-th measurement of party $j$ is denoted by $M_{j}^{i_{j}}, j=$ $1, \ldots, n, i_{j}=1, \ldots, m$. The correlation function equality with respect to this system can be taken as,

$$
E_{i_{1} \ldots i_{n}} E_{i_{1}^{\prime} \ldots i_{n}^{\prime}}=P\left\{E_{i_{1} \ldots i_{n}} E_{i_{1}^{\prime} \ldots i_{n}^{\prime}}\right\}
$$

where $E_{i_{1} \ldots i_{n}}=\left\langle M_{1}^{i_{1}} \ldots M_{n}^{i_{n}}\right\rangle$ and $P$ represent the exchange of any number of measurement of observables between two correlation function $i_{1} \ldots i_{n}: i_{1}^{\prime} \ldots i_{n}^{\prime}$. Equality (9) can easily be demonstrated to be true for separable pure state. We remark that, in general, we don't have to restrict the number of correlation function to be two in both sides. The simplest one of all those equalities (9) is $\left\langle A_{i} B_{j}\right\rangle=\left\langle A_{i}\right\rangle\left\langle B_{j}\right\rangle$, which can be obtained by choosing $A_{k}=I$ and $B_{l}=I$ in (2).
This equality can also be expressed as $p\left(A_{i}=a, B_{j}=b\right)=$ $p\left(A_{i}=a\right) p\left(B_{j}=b\right)$, where $a$ and $b$ are outcomes of Alice's and Bob's measurement. The failure of this equality in entangled pure state suggest that local measurement may act as a detection for the entangled states.

It is obvious that pure separable state for multipartite system does not violate this equality. While quantum entangled state will have a violation. As an example, we assume each one of $n$ parties can choose between two measurements, $i_{j}=1,2$. $P$ represents exchange first $r$ measurement between two correlation function, which is

$$
P\left\{E_{i_{1} \ldots i_{n}} E_{i_{1}^{\prime} \ldots i_{n}^{\prime}}\right\}=E_{i_{1}^{\prime} \ldots i_{r}^{\prime} i_{r+1} \ldots i_{n}} E_{i_{1} \ldots i_{r} i_{r+1}^{\prime} \ldots i_{n}^{\prime}} .
$$

The violation can be derived immediately when system is prepared in entangled state. We define quadrature operators [15],

$$
\begin{aligned}
& M_{j}^{1}=\hat{a}_{j}+\hat{a}_{j}^{\dagger}, \\
& M_{j}^{2}=\hat{a}_{j} e^{-i \phi_{j}}+\hat{a}_{j}^{\dagger} e^{i \phi_{j}},
\end{aligned}
$$

where $\phi_{j}$ is local measurement phase parameters with respect to observer $j$. If the first $r$ observers set their local phase parameters are $+\pi / 2$, and the remaining set theirs as $-\pi / 2$. Measurement is performed in quantum state,

$$
|\Psi\rangle=\sin \theta|0\rangle^{r}|1\rangle^{n-r}+\cos \theta e^{i \phi}|1\rangle^{r}|0\rangle^{n-r},
$$

which is the superposition of $r$ modes ( $n-r$ modes) all occupying one (no) photon and vice versa. Then we find,

$$
\begin{aligned}
E_{1 \cdots 1} E_{2 \cdots 2}= & \sin ^{2} 2 \theta \cos \phi \cos (\phi-n \pi / 2) \\
E_{1 \cdots 12 \cdots 2} E_{2 \cdots 21 \cdots 1}= & \sin ^{2} 2 \theta \cos [\phi-(n-r) \pi / 2] \\
& \times \cos (\phi-r \pi / 2)
\end{aligned}
$$

Choose these parameters appropriately, one can always find that two sides of Eq. (9) does not equal. When $n=2$ and $r=1$, we recover the previous result. We remark that like the $n=2$ case, general mixed separable state does not always satisfy the equality.

We now study the CFRD inequality, which is a Bell-type inequality for continuous variables with respect to $n$ parties and each party can carry out two dichotomic measurements [15]. It takes the form,

$$
\left|\left\langle\prod_{j=1}^{n}\left[M_{j}^{1}+i M_{j}^{2}\right]\right\rangle\right|^{2} \leq\left\langle\prod_{j=1}^{n}\left[\left(M_{j}^{1}\right)^{2}+\left(M_{j}^{2}\right)^{2}\right]\right\rangle,
$$

where $M_{j}^{i_{j}}$ are $i_{j}$-th measurement choice with respect to observer $j$ as denoted before.

First let us consider the simplest $n=2$ case. To derive the inequality, it is assumed that, $E_{11} E_{22}=E_{12} E_{21}$, which is exact the condition (2), where $E_{i_{1} i_{2}}^{2}=\left\langle M_{1}^{i_{1}} M_{2}^{i_{2}}\right\rangle^{2}$, see [15]. So the CFRD inequality is based on the fact that all measurement have nonnegative variance, that is,

$$
\left\langle\left(M_{1}^{i_{1}}\right)^{2}\left(M_{2}^{i_{2}}\right)^{2}\right\rangle-\left\langle M_{1}^{i_{1}} M_{2}^{i_{2}}\right\rangle^{2} \geq 0
$$


Thus the CFRD inequality (15) can be violated only for case that (2) is not satisfied. Thus, the violation of equation, $E_{11} E_{22} \neq E_{12} E_{21}$, is more powerful than the violation of CFRD inequality to detect the entangled state. As we have shown, this property can detect all entangled state of form (12).

The CFRD inequality is proposed in Ref.[15], they found that the inequality is violated for case $n \geq 10$ for state (12). Then in Ref.[20], the violation condition is extended to case $n \geq 5$. Additionally if we apply the method of [18], the violation case can be extended to $n \geq 3$, see next section for detail. On the other hand, in this paper, we find the violation of Eq.(2) can detect all entangled state of form (12). Thus we show that the violation of Eq.(2) is a necessary condition for the violation of CFRD inequality. We remark that the entangled state can be detected by a different approach by using two classes of multimode Bell inequalities proposed in Ref.[21] for case $n \geq 2$. Here we present a much simpler method which can detect all cases $n \geq 2$.

The general case seems straightforward. For simplicity, we denote $M=\left\langle\prod_{j=1}^{n}\left[M_{j}^{1}+i M_{j}^{2}\right]\right\rangle$. The real part of $M$ is the sum of $n$ partite correlation function which only contain even numbers of measurement observable with $i_{j}=2$ and the imaginary part of $M$ contain odd numbers. We always find that cross term of correlation function appears in $(\operatorname{Re} M)^{2}$ and its opposite counterpart which generally have form (10) appears in $(\operatorname{Im} M)^{2}$. For separable state pure state, they vanish which leave us with terms of all square of correlation function of $n$ parties, which is $M=M^{\prime} \equiv$ $\sum_{i_{1}, \cdots, i_{n}=1,2}\left\langle M_{1}^{i_{1}} \cdots M_{n}^{i_{n}}\right\rangle^{2}$. This is always less than or equal to $\sum_{i_{1}, \cdots, i_{n}=1,2}\left\langle\left(M_{1}^{i_{1}}\right)^{2} \cdots\left(M_{n}^{i_{n}}\right)^{2}\right\rangle$. As has been demonstrated in $n=2$ case, the fact $M^{\prime}$ is always less than or equal to the right side of (15) leads us to know, as expected, all the violation of CFRD inequality comes from the violation of Eq.(2) and its generalization.

\section{VIOLATION OF CFRD INEQUALITY}

Here we show that the violation of CFRD inequality can be extended to case $n \geq 3$ for state (12). We introduce the notations,

$$
\begin{aligned}
& s_{z}=\sum_{k=0}^{\infty}[|2 k+1\rangle\langle 2 k+1|-| 2 k\rangle\langle 2 k|], \\
& s_{-}=\sum_{k=0}^{\infty}|2 k\rangle\langle 2 k+1|=\left(s_{+}\right)^{\dagger} .
\end{aligned}
$$

Those operators can be used to find the violation of $\mathrm{CHSH}$ inequality for TMSS [6], see Ref.[18]. The measurement operators in (15) can be defined as,

$$
\begin{aligned}
& M_{j}^{1}=s_{-}^{j}+s_{+}^{j}, \\
& M_{j}^{2}=s_{-}^{j} e^{-i \phi_{j}}+s_{+}^{j} e^{i \phi_{j}} .
\end{aligned}
$$

For state (12), similar as in Ref.[15], let the first $r$ phase parameters $\phi_{j}=\pi / 2$, the last $n-r$ phase parameters $\phi_{j}=-\pi / 2$. So for $j \leq r, M_{j}^{1}+i M_{j}^{2}=2 s_{-}^{j}$, and for $j>r, M_{j}^{1}+i M_{j}^{2}=2 s_{+}^{j}$, also we have $\left(M_{j}^{1}\right)^{2}+\left(M_{j}^{2}\right)^{2}=$ $2\left(s_{-}^{j} s_{+}^{j}+s_{+}^{j} s_{-}^{j}\right)$. Now let us consider CFRD inequality (15), we find,

$$
\begin{aligned}
& \text { l.h.s }=2^{2 n}\left|\left\langle s_{-}^{1} \ldots s_{-}^{r} s_{+}^{r+1} \ldots s_{+}^{n}\right\rangle\right|^{2} \\
& \text { r.h.s }=2^{n}\left\langle\left(s_{-}^{1} s_{+}^{1}+s_{+}^{1} s_{-}^{1}\right) \ldots\left(s_{-}^{n} s_{+}^{n}+s_{+}^{n} s_{-}^{n}\right)\right\rangle .
\end{aligned}
$$

With the exact form of (12), we find,

$$
\begin{aligned}
& \text { l.h.s }=2^{2 n} \cos ^{2} \theta \sin ^{2} \theta, \\
& \text { r.h.s }=2^{n} .
\end{aligned}
$$

We then let $\cos ^{2} \theta=\sin ^{2} \theta=1 / 2$, the l.h.s achieves the maximal point $2^{2 n} / 4$. Then one may find that when $n=3$,

$$
\text { l.h.s }=16>\text { r.h.s }=8,
$$

The CFRD inequality (15) is violated, and the violation is true for cases $n>3$. Then here the violation region is for $n \geq 3$ which extends the results in Refs. [15, 20] in which the violation cases are: $n \geq 10$ and $n \geq 5$, respectively.

\section{CONCLUSION}

Much of recent study of entanglement focuses on Bell type inequalities, both theoretically and experimentally. In this paper, we propose a simple equality by local measurement to detect the entangled state. It provides a much simpler criterion to detect entangled state for discrete and CV systems, additionally it may have the advantage that the violation is not affected by depolarizing noise of decoherence. In addition, it is the origination of the violation of CFRD inequality. Actually, all entangled state can be detected by this condition. Besides of CV systems of optics, it can also be easily checked in some other physical systems, for example, in superconducting systems, see Ref. [22] for a review. The proposed equation is simple and powerful, and is an important complement to Bell inequalities. Depending on question and system, we may choose to find the violation of either Bell inequalities or this simple equation.

This work is supported by NSFC(10931006, 10975180, 10974247, 11075126), “973” program (2010CB922904).

[1] A. Einstein, B. Podolsky, and N. Rosen, Phys. Rev. 47, 777 (1935).

[2] J.S. Bell, Physics (Long Island City, N. Y.) 1, 195 (1964).

[3] J.S. Bell, Rev. Mod. Phys. 38, 447 (1966).

[4] J.F. Clauser, M.A. Horne, A. Shimony, and R.A. Holt, Phys. Rev. Lett. 23, 880 (1969).

[5] N.D. Mermin, Phys. Rev. Lett. 65, 1838 (1990); Phys. Rev. Lett. 65, 3373 (1990); Rev. Mod. Phys. 65, 803 (1990). 
[6] D. Collins, N. Gisin, N. Linda, S. Massar, and S. Popescu, Phys. Rev. Lett. 88, 040404 (2002).

[7] M. Żukowski and Č. Brukner, Phys. Rev. Lett. 88, 210401 (2002).

[8] W. Son, J. Lee, and M. S. Kim, Phys. Rev. Lett. 96, 060406 (2006).

[9] A. Aspect, P. Grangier, and G. Roger, Phys. Rev. Lett. 49, 91 (1982).

[10] G. Weihs, J. Jennewein, C. Simon, H. Weinfurter, A. Zeilinger, Phys. Rev. Lett. 81, 5039 (1998).

[11] M. A. Rowe et al, Nature (London) 409, 791 (2001).

[12] S. Pironio et al, Nature (London) 461, 1021 (2010).

[13] S. Kochen and E. P. Specker, J. Math. Mech. 17, 59 (1967).

[14] M. Genovese, Phys. Rep. 413, 319 (2005).

[15] E.G. Cavalcanti, C.J. Forster, M.D. Reid, and P.D. Drummond,
Phys. Rev. Lett. 99, 210405 (2007).

[16] N. Gisin, Phys. Lett. A 154, 201 (1991); N. Gisin and A. Peres, Phys. Lett. A 162, 15 (1992).

[17] J. L. Chen, C. F. Wu, L. C. Kwek, and C. H. Oh, Phys. Rev. Lett. 93, 140407 (2004).

[18] Z.-B. Chen, J.-W. Pan, G. Hou, and Y.-D. Zhang, Phys. Rev. Lett. 88, 040406 (2002).

[19] R. F. Werner, Phys. Rev. A 40, 4277 (1989).

[20] Q.Y. He, E.G. Cavalcanti, M.D. Reid, and P.D. Drummond, Phys. Rev. Lett. 103, 180402 (2009).

[21] S.W. Ji, J. Kim, H.W. Lee, M.S. Zubairy, and H. Nha, Phys. Rev. Lett. 105, 170404 (2010).

[22] J. Q. You and F. Nori, Nature 474, 589 (2011). 